\title{
A New Approach for Pure Kinematical and Reduced Kinematical Determination of a LEO Orbit based on GNSS Observations
}

Akbar Shabanloui

Session: Gravity 2.2

IAG 2009

31th August 2009, Buenos Aires 


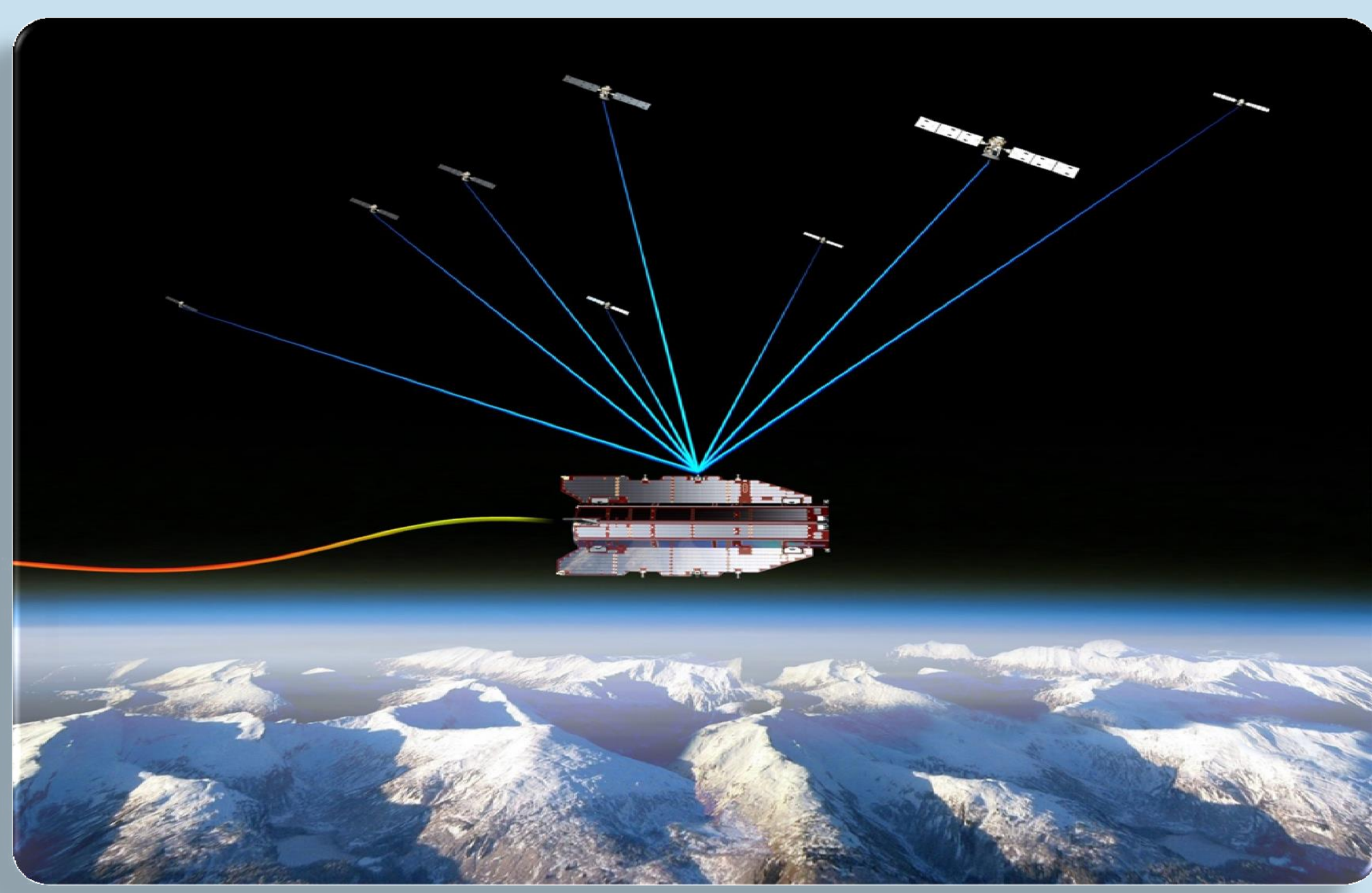




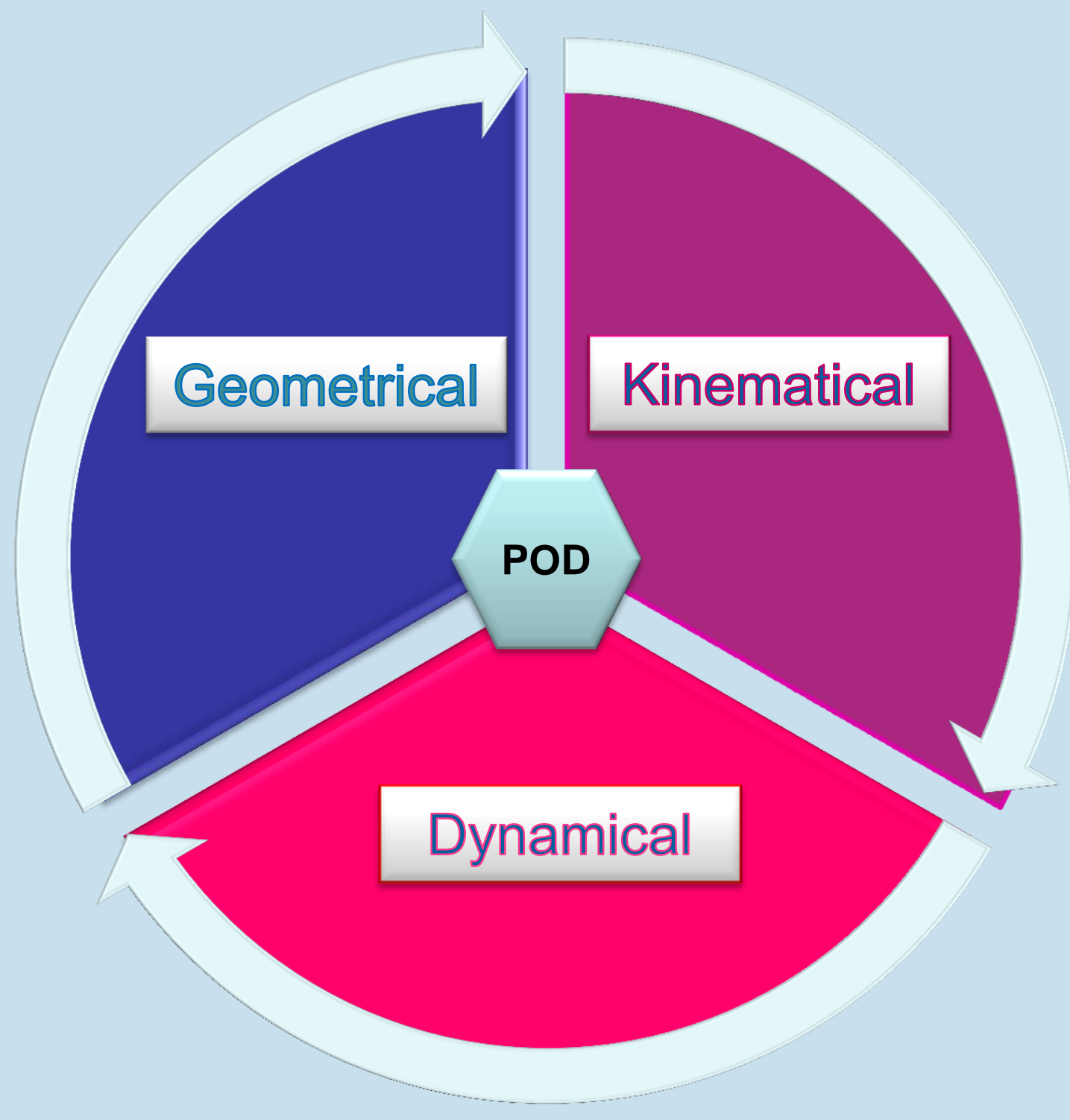




\section{Precise Orbit Determination (POD) methods}

$>$ Geometrical POD : point-wise, positions != Kinematical POD

> Kinematical POD : continous, positions, velocities and accelerations

> Dynamical POD : continous, positions, velocities and accelerations based on force function information

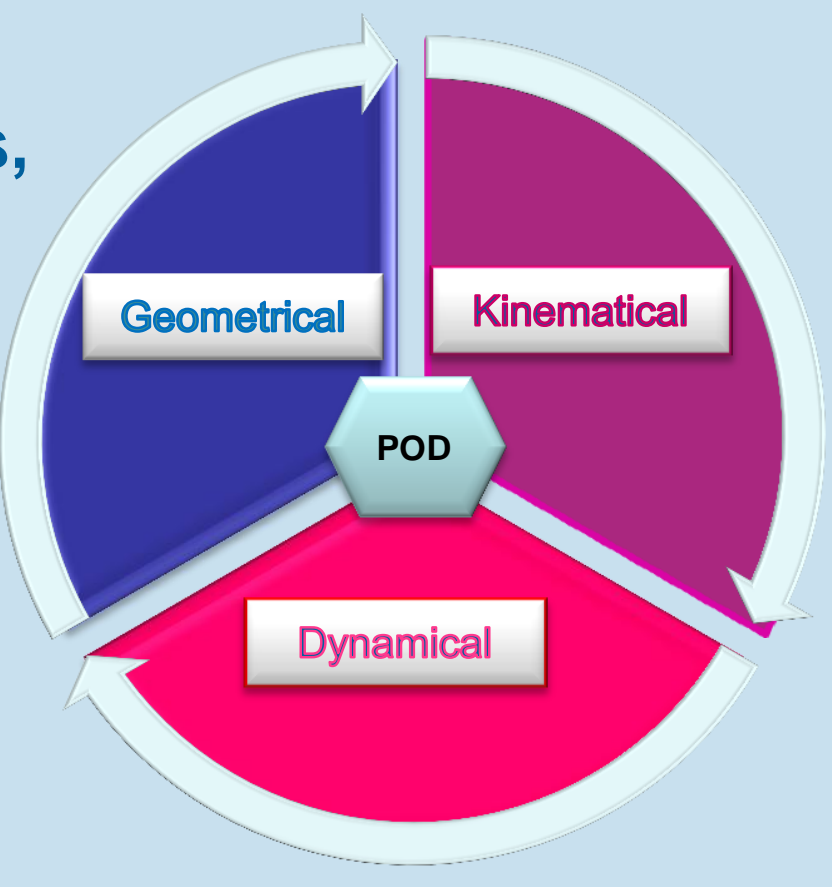




\section{Kinematical Precise Orbit Determination (KPOD)}

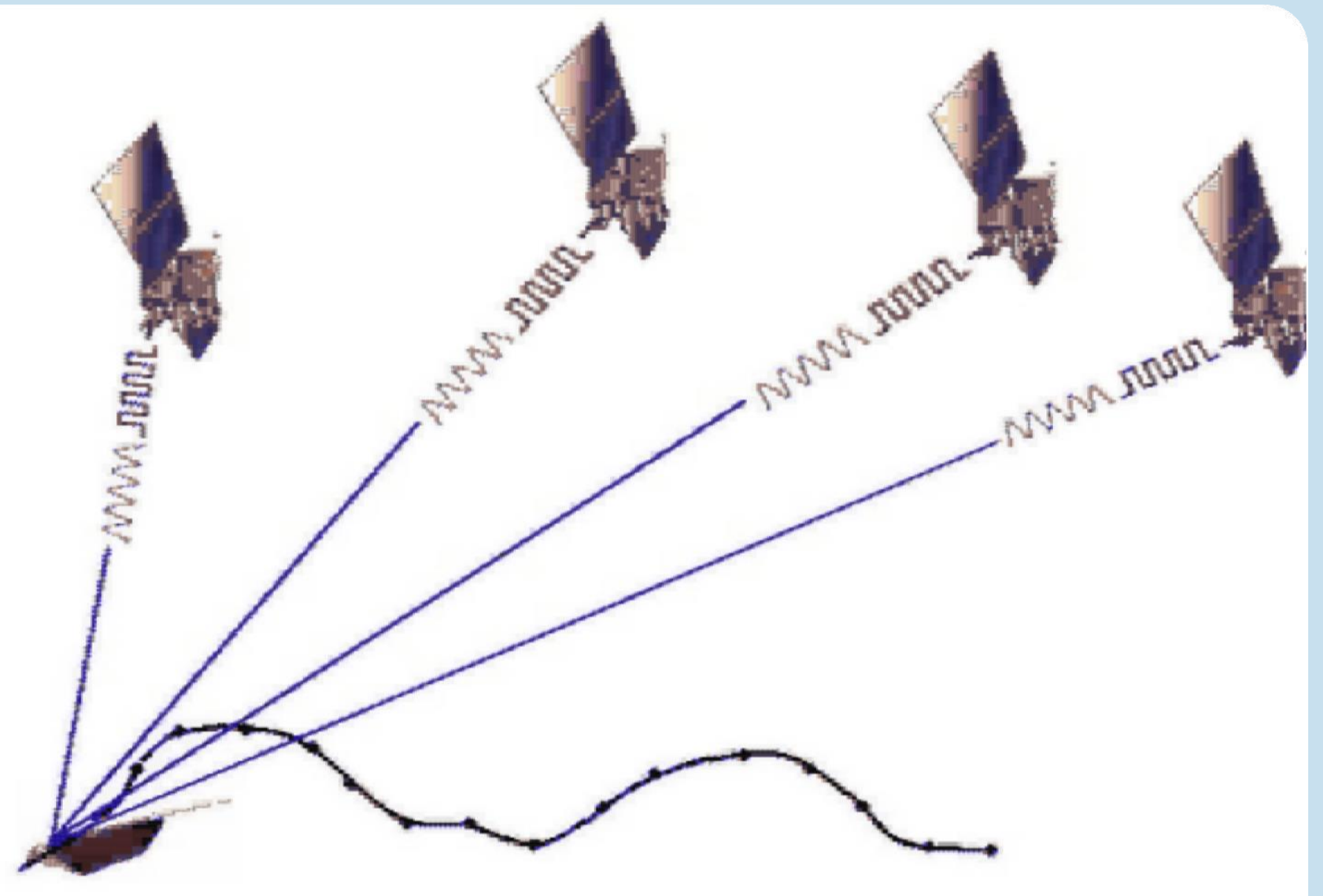




\section{Processing procedures}

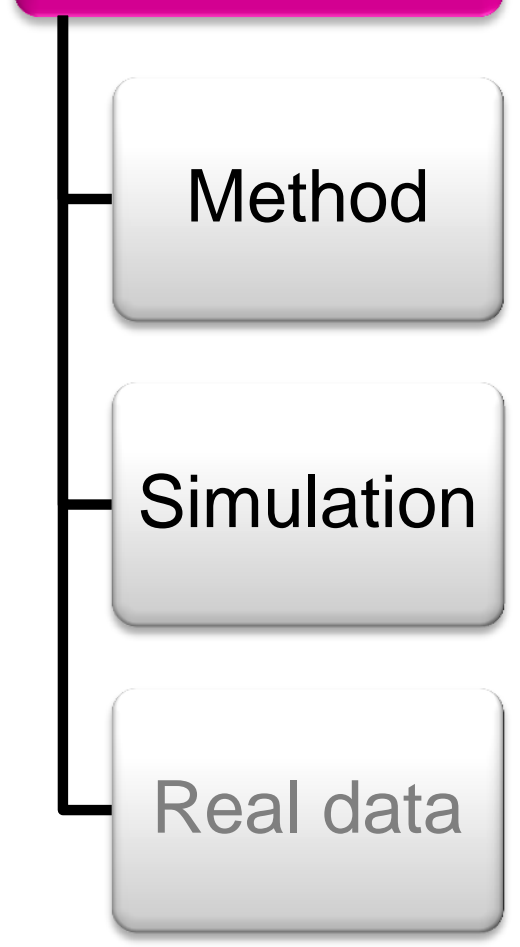




\section{Processing procedures}

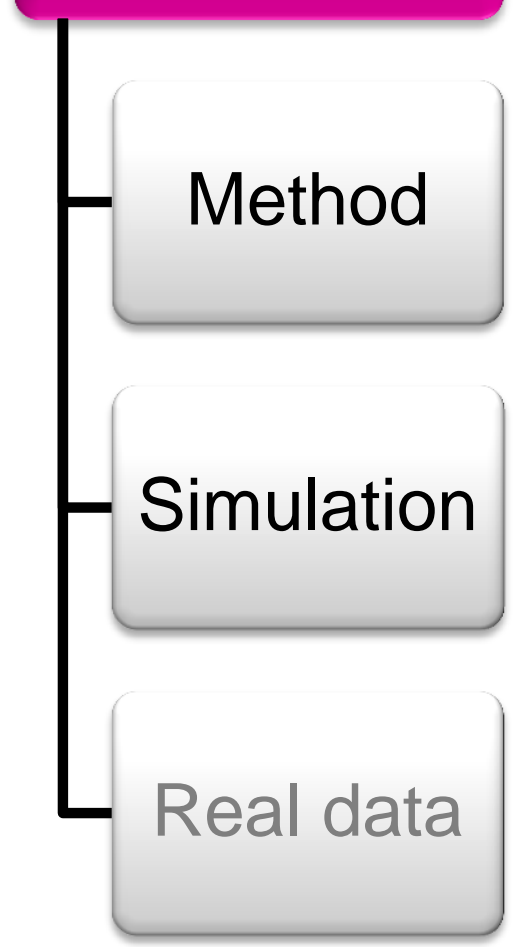




\section{Kinematical POD - methods}

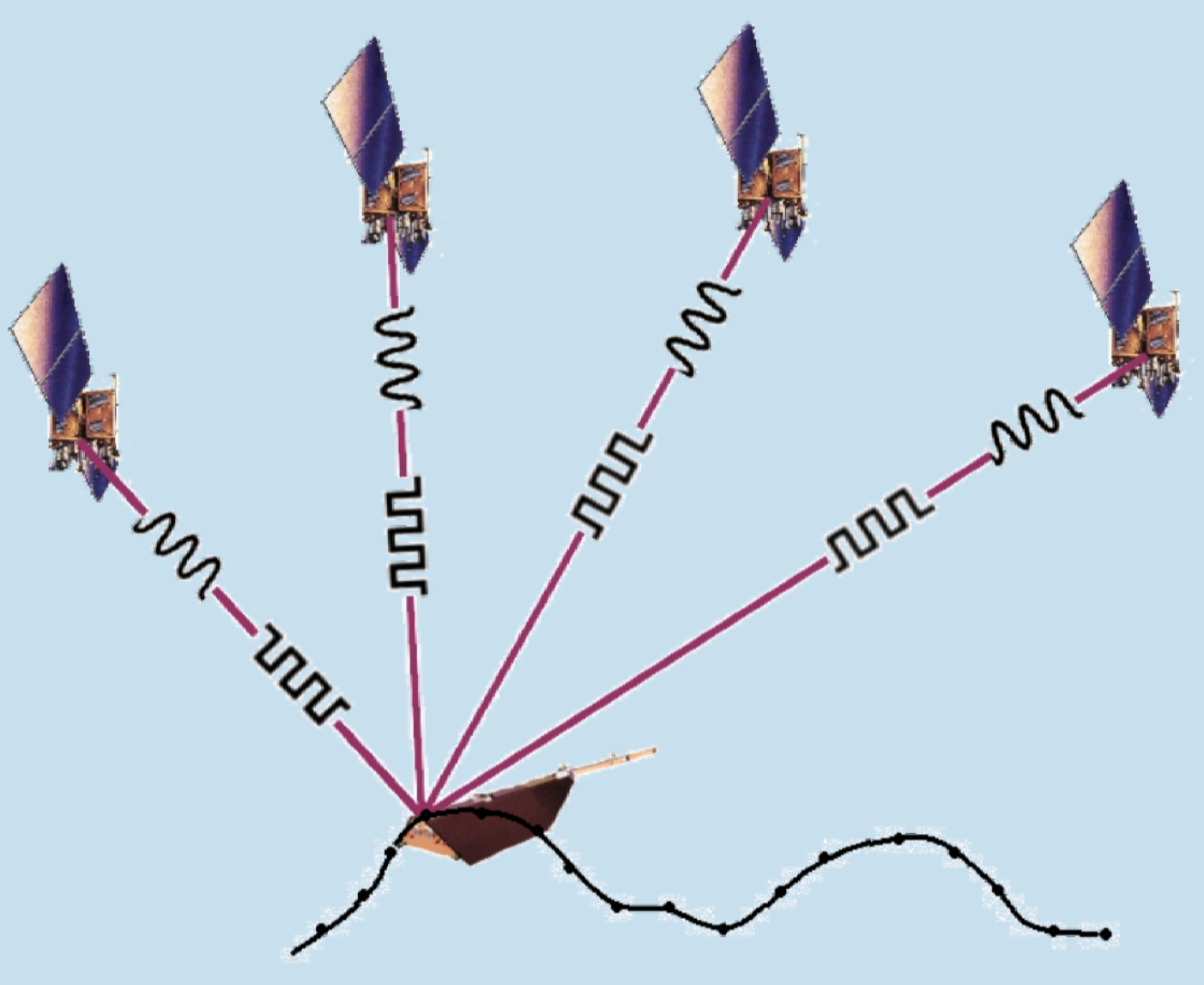




\section{Kinematical POD - methods}

Equation of motion w.r.t self-adjoint differential operator:

$$
L(\mathbf{r}(t))=\mathbf{a}(t ; \mathbf{r}, \dot{\mathbf{r}})
$$

corresponding Fredholm's integral equation:

Reference motion Integral kernel

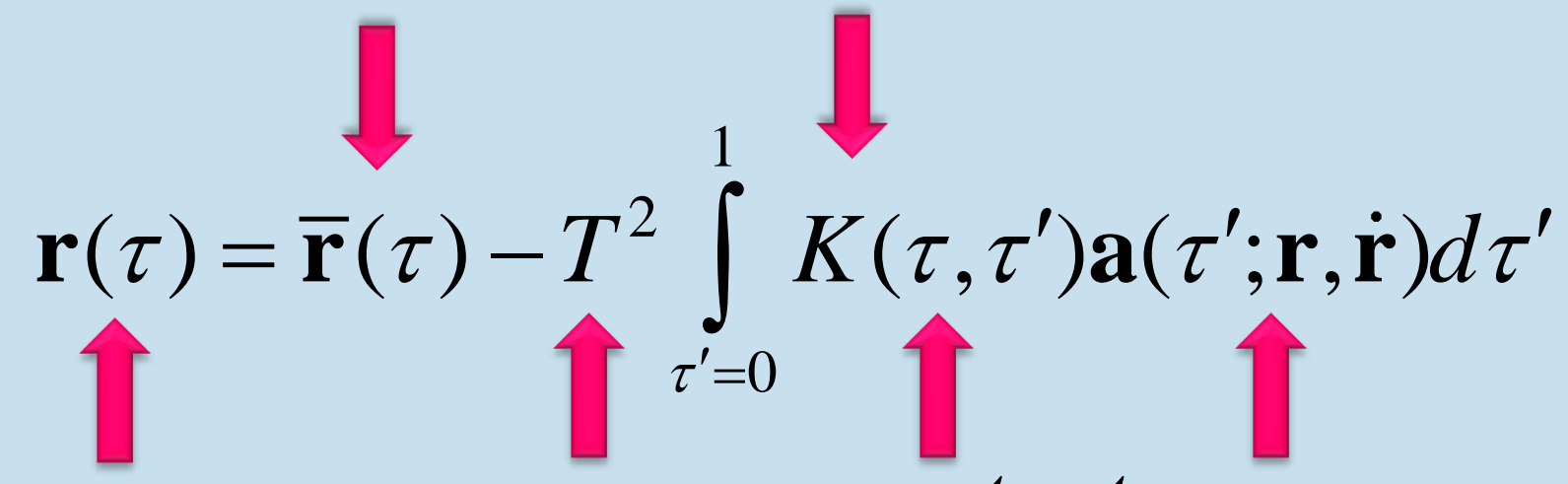

Position $\quad T=t_{B}-t_{A} \quad \tau=\frac{t-t_{A}}{T} \quad$ Force function

$$
L(\overline{\mathbf{r}}(\tau))=0
$$




\section{Short arc representation - ellipse mode}

A satellite short arc:

$$
\mathbf{r}(\tau)=\overline{\mathbf{r}}(\tau)+\mathbf{d}(\tau)=\overline{\mathbf{r}}(\tau)+\sum_{v=1}^{\infty} \mathbf{d}_{v} \sin (v \pi \tau)
$$

elliptical reference motion:

$$
\overline{\mathbf{r}}(\tau)=\mathbf{r}_{A} \frac{\sin \mu(1-\tau)}{\sin \mu}+\mathbf{r}_{B} \frac{\sin \mu \tau}{\sin \mu}
$$

difference function:

$\mathbf{d}(\tau)=\sum_{\nu=1}^{\infty} \mathbf{d}_{\nu} \sin (v \pi \tau)$ 


\section{Short arc representation - Fourier series}

A satellite short arc can be represented:

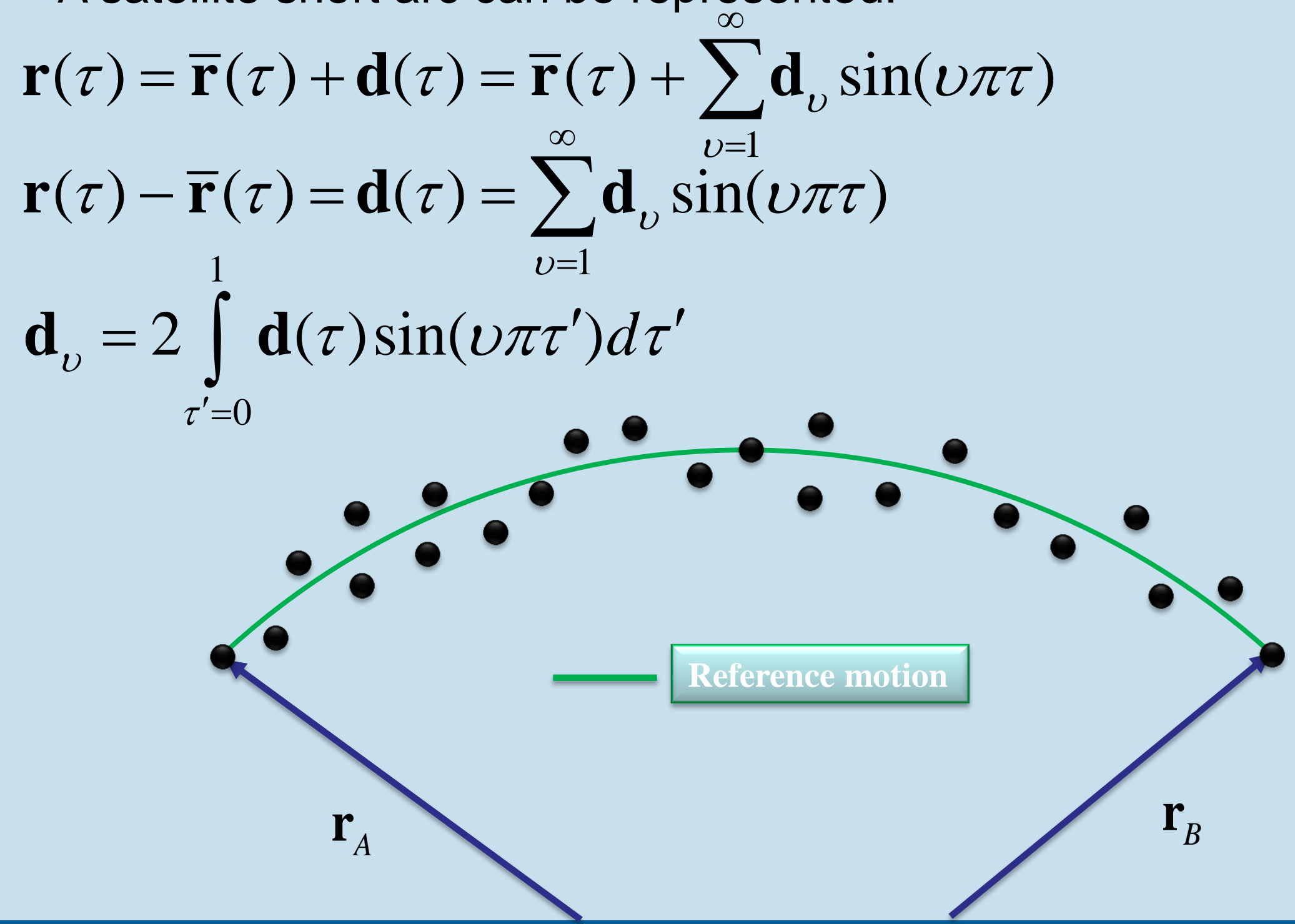




\section{Short arc representation - Fourier series}

A satellite short arc can be represented:

$$
\begin{aligned}
& \mathbf{r}(\tau)-\overline{\mathbf{r}}(\tau)=\mathbf{d}(\tau)=\sum_{v=1}^{\infty} \mathbf{d}_{v} \sin (v \pi \tau) \\
& \mathbf{d}_{v} \approx \frac{2}{N_{e}+1} \sum_{e=1}^{N_{e}} \mathbf{d}\left(\tau_{e}\right) \sin \left(\frac{v \pi e}{N_{e}+1}\right)
\end{aligned}
$$

$\mathbf{d}\left(\tau_{e}\right) \quad$ with $\left.\quad \tau_{e}=\frac{e}{N_{e_{e}}+1}, \quad e=1, \ldots, N_{e} \quad \tau_{e} \in\right] 0,1[$

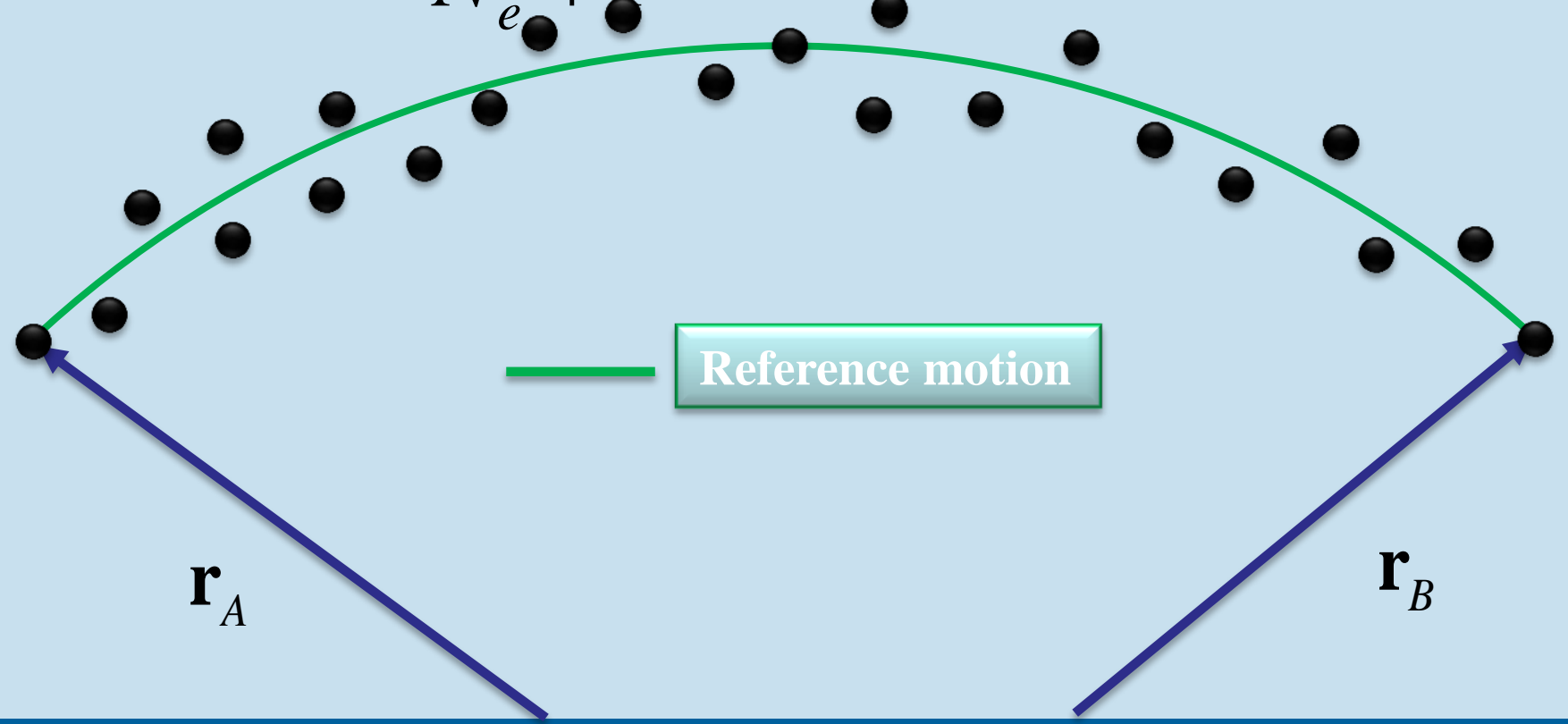




\section{Fourier analysis - ellipse mode}

- $d_{v, x}-d_{v, y}-d_{v, z}$

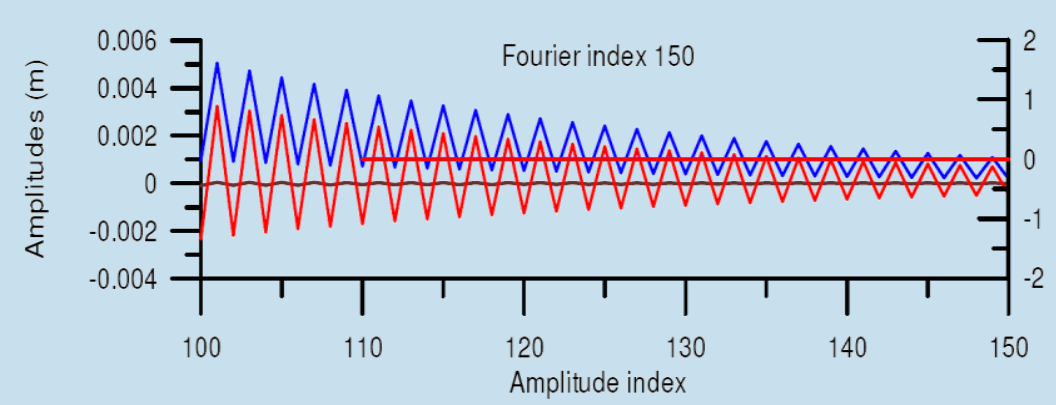

- $d_{\mathrm{x}} \quad \Delta \mathrm{d}_{\mathrm{y}} \quad \sim \mathrm{d}_{\mathrm{z}}$

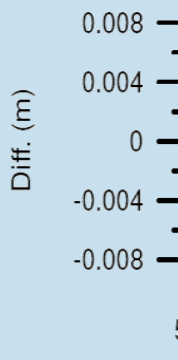

Fourier index 150
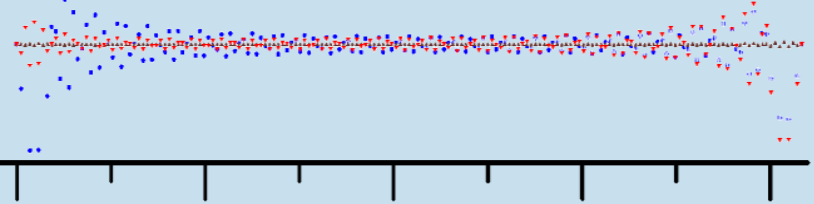

51743.75
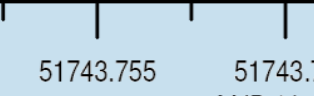

MJD (days)

\section{Amplitudes}

Remainders 


\section{Short arc representation - Euler-Bernoulli}

A satellite short arc can be represented:

with Fourier amplitudes:

$$
\mathbf{r}(\tau)=\overline{\mathbf{r}}(\tau)+\sum_{v=1}^{\infty} \mathbf{d}_{v} \sin (v \pi \tau)
$$

$$
\mathbf{d}_{v}=2 \int_{\tau^{\prime}=0}^{1} \mathbf{d}(\tau) \sin \left(v \pi \tau^{\prime}\right) d \tau^{\prime}
$$

Fourier series amplitudes:

$$
\begin{aligned}
\mathbf{d}_{v} & =\sum_{j=1}^{J} \frac{2(-1)^{j+1}}{(v \pi)^{2 j+1}}\left[(-1)^{v} \mathbf{d}^{[2 j]}(1)-\mathbf{d}^{[2 j]}(0)\right]+ \\
& +\beta \frac{2}{(v \pi)^{2 J+1}} \int_{\tau^{\prime}=0}^{1} \mathbf{d}^{[2 J+2]}\left(\tau^{\prime}\right) \sin \left(v \pi \tau^{\prime}\right) d \tau^{\prime}
\end{aligned}
$$




\section{Short arc representation - Euler-Bernoulli}

$$
\begin{gathered}
\mathbf{d}_{F}^{\infty}=\mathbf{d}(\tau)=\sum_{\nu=1}^{\infty} \mathbf{d}_{v} \sin (v \pi \tau)= \\
=\sum_{j=1}^{\infty} \mathbf{e}_{2 j} E_{2 j}(\tau)+\sum_{j=1}^{\infty} \mathbf{b}_{2 j+1} B_{2 j+1}(\tau)=\mathbf{d}_{P}^{\infty}
\end{gathered}
$$

A satellite short arc can be represented:

$$
\sum_{v=1}^{\infty} \mathbf{d}_{v} \sin (v \pi \tau)=\sum_{j=1}^{\infty} \mathbf{e}_{2 j} E_{2 j}(\tau)+\sum_{j=1}^{\infty} \mathbf{b}_{2 j+1} B_{2 j+1}(\tau)
$$




\section{Determination of Euler-Bernoulli coefficients}

A satellite short arc can be represented with the Euler-Bernoulli term up to degree $J$ as:

$$
\mathbf{r}(\tau)-\overline{\mathbf{r}}(\tau)=\mathbf{d}(\tau) \approx \sum_{j=1}^{J} \mathbf{e}_{2 j} E_{2 j}(\tau)+\sum_{j=1}^{J} \mathbf{b}_{2 j+1} B_{2 j+1}(\tau)
$$

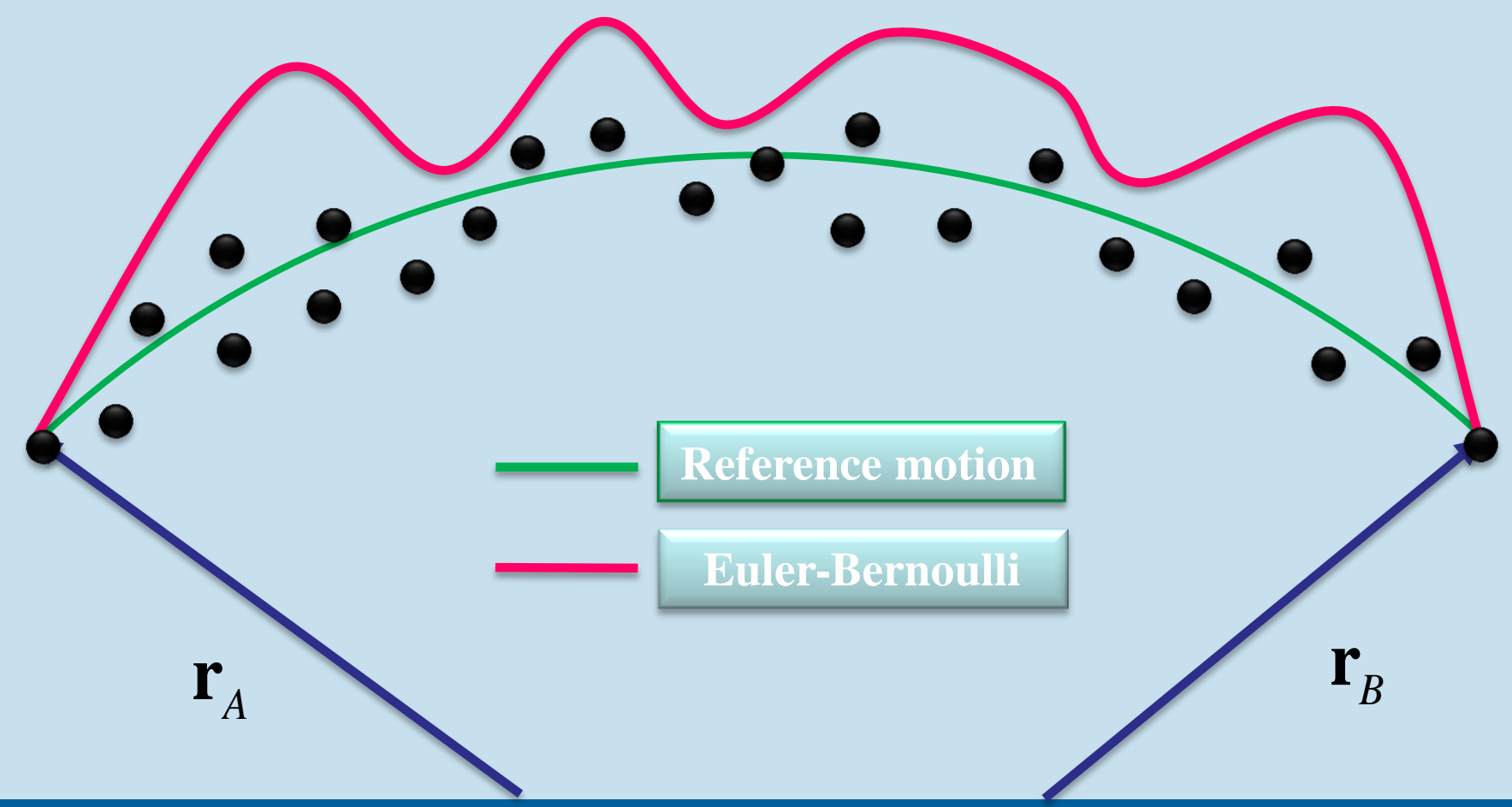




\section{Determination of Euler-Bernoulli coefficients}
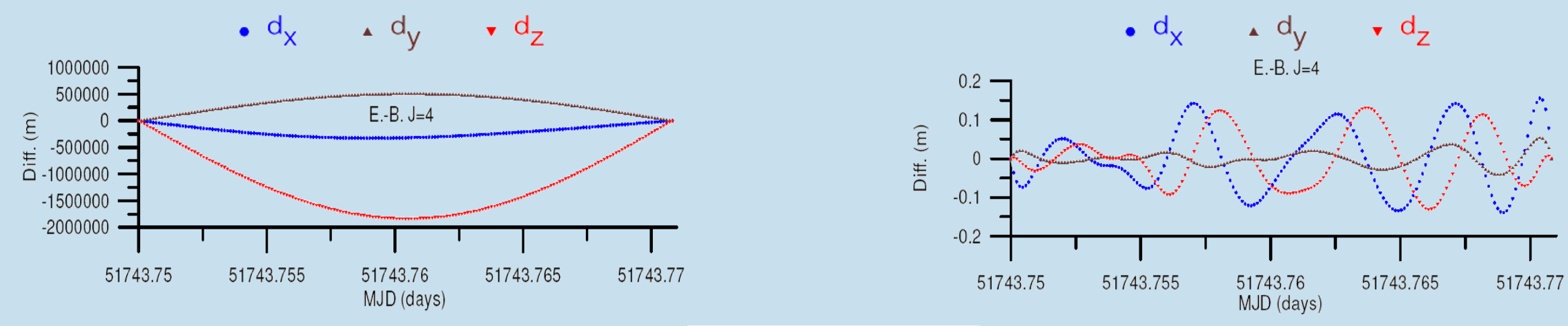

ellipse mode

E.-B terms

Remainders 


\section{Short arc representation}

LEO orbit can be represented as:

\section{Gibbs effect!}

$$
\mathbf{r}(\tau)=\overline{\mathbf{r}}(\tau)+\mathbf{d}(\tau)=\overline{\mathbf{r}}(\tau)+\sum_{v=1}^{n} \mathbf{d}_{v} \sin (\vartheta \pi \tau)
$$

or

Precision!

$$
\mathbf{r}(\tau)=\overline{\mathbf{r}}(\tau)+\mathbf{d}(\tau)=\overline{\mathbf{r}}(\tau)+\sum_{j=1}^{J} \mathbf{e}_{2 j} E_{2 j}(\tau)+\sum_{j=1}^{J} \mathbf{b}_{2 j+1} B_{2 j+1}(\tau)
$$

\section{Solution?}

fast

\section{convergence!}

$\mathbf{r}(\tau)=\overline{\mathbf{r}}(\tau)+\sum_{j=1}^{J} \mathbf{e}_{2 j} E_{2 j}(\tau)+\sum_{j=1}^{J} \mathbf{b}_{2 j+1} B_{2 j+1}(\tau)+\sum_{v=1}^{\bar{n}} \overline{\mathbf{d}}_{v} \sin (v \pi \tau)$ 


\section{Kinematical POD - ellipse mode, J=4}
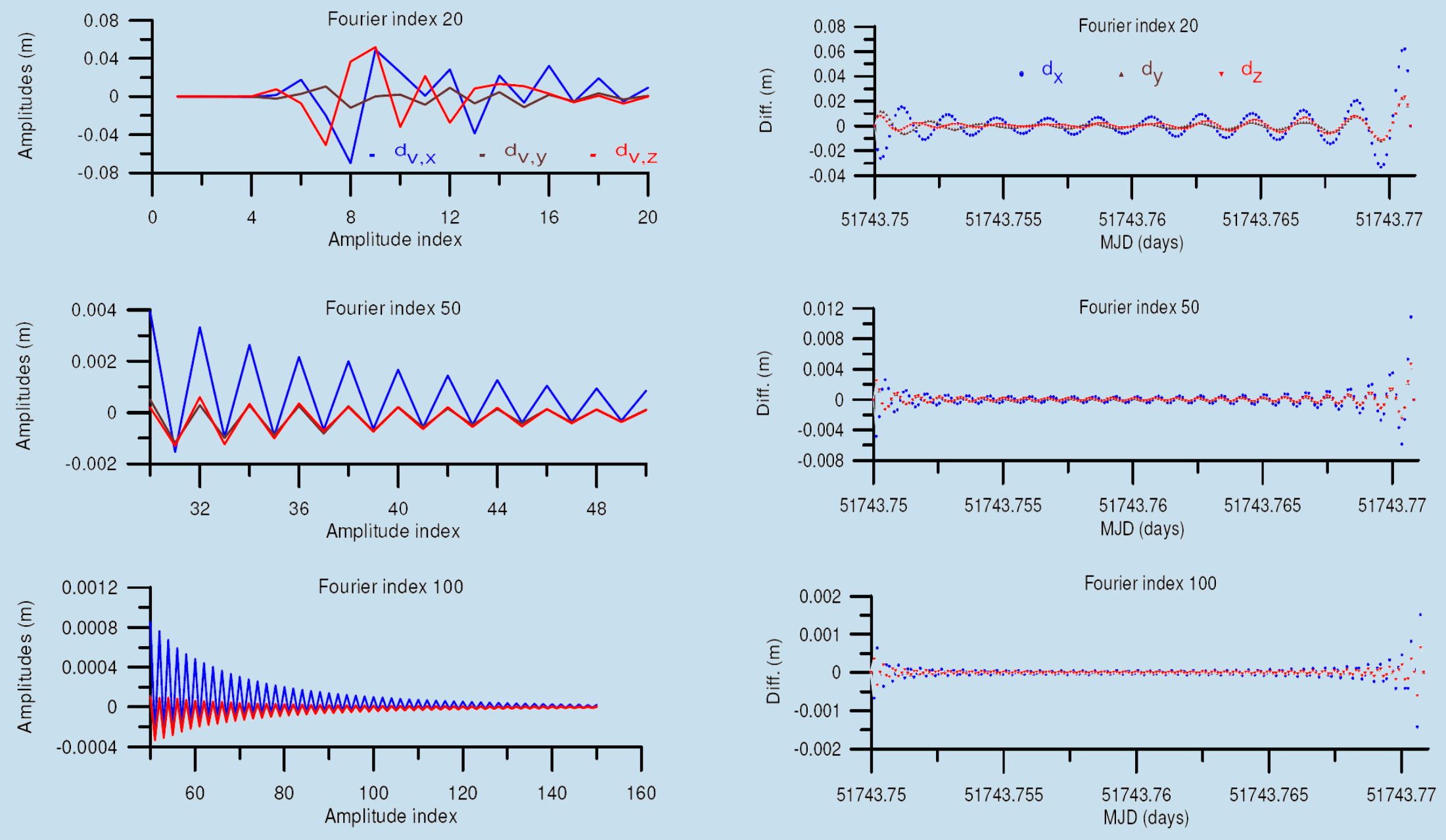

\section{Amplitudes}

Remainders 


\section{Processing procedures}

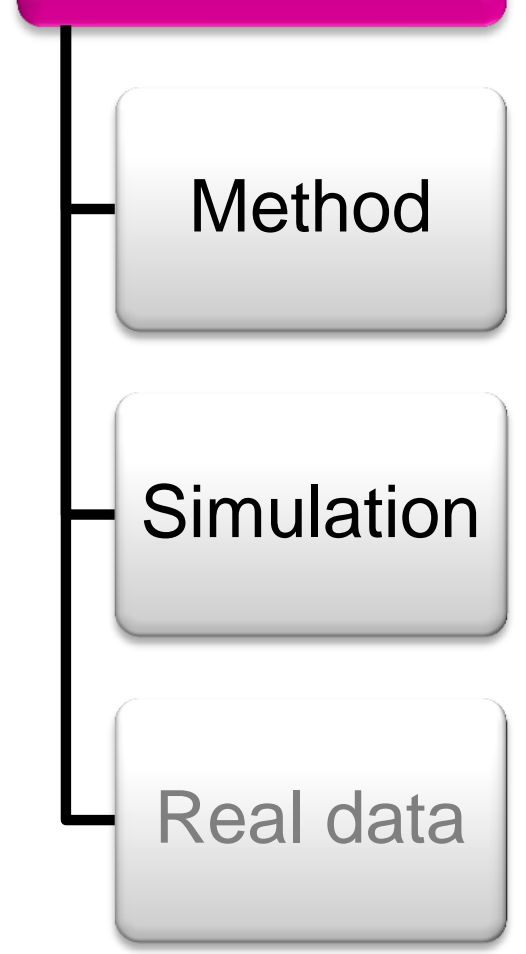




\section{Kinematical POD - simulation $(2 \mathrm{~cm} \text { noise SST })_{\text {niversitätbonn }}$}

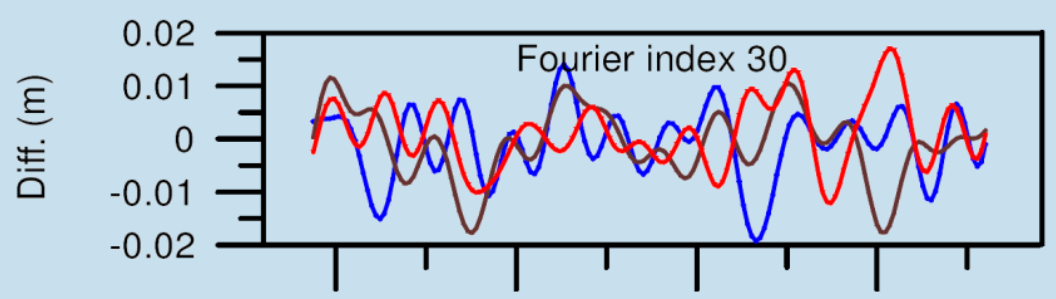

51742.335 51742.34 51742.345 51742.35 MJD (days)

\section{Position differences}

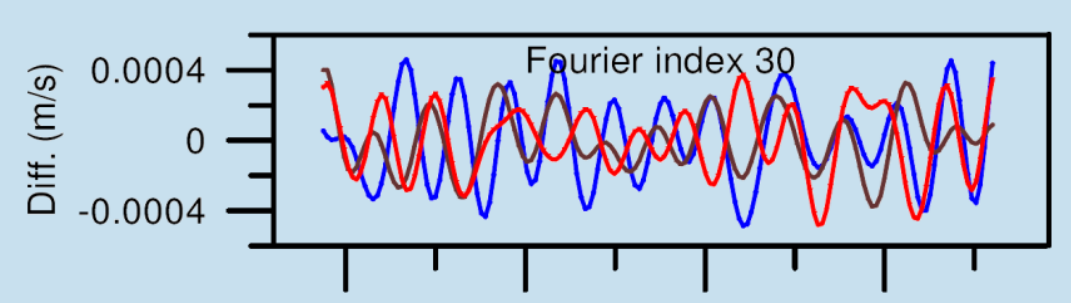

51742.33551742 .3451742 .34551742 .35 MJD (days)

\section{Velocity differences}

RMS

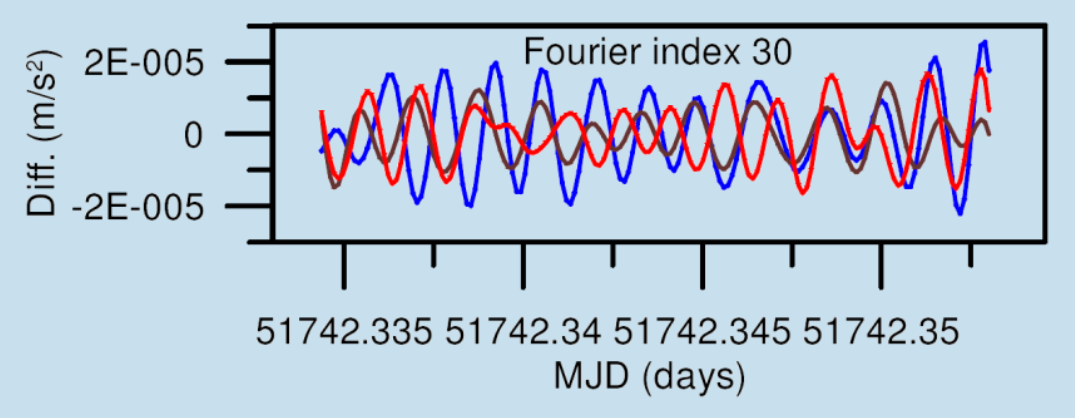

Acceleration differences

\begin{tabular}{|c|c|c|c|}
\hline index & Pos. $(\mathrm{m})$ & Vel. $(\mathrm{m} / \mathrm{s})$ & Acc. $\left(\mathrm{m} / \mathrm{s}^{2}\right)$ \\
\hline 20 & 0.012644 & 0.000353 & 0.000012 \\
\hline 30 & 0.010717 & 0.000397 & 0.000018 \\
\hline 40 & 0.011997 & 0.000463 & 0.000025 \\
\hline 59 & 0.014737 & 0.000941 & 0.000077 \\
\hline
\end{tabular}




\section{Processing procedures}

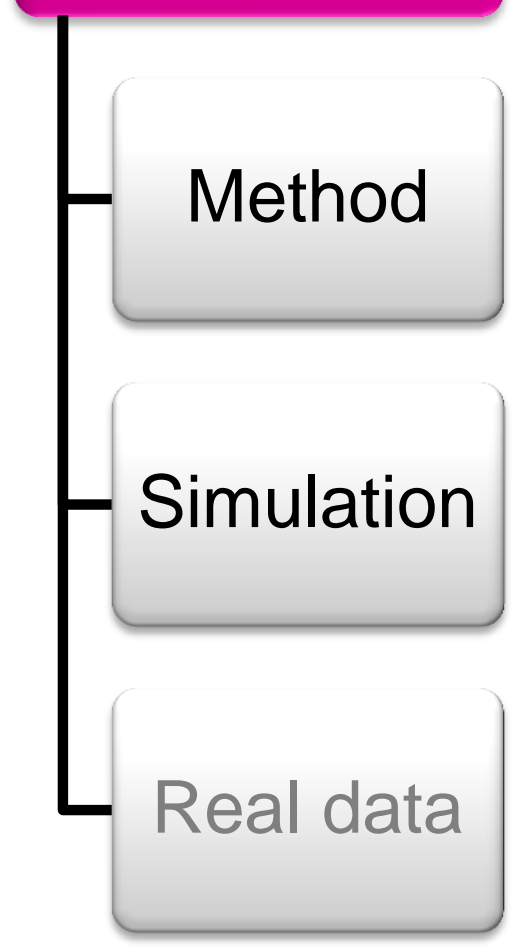




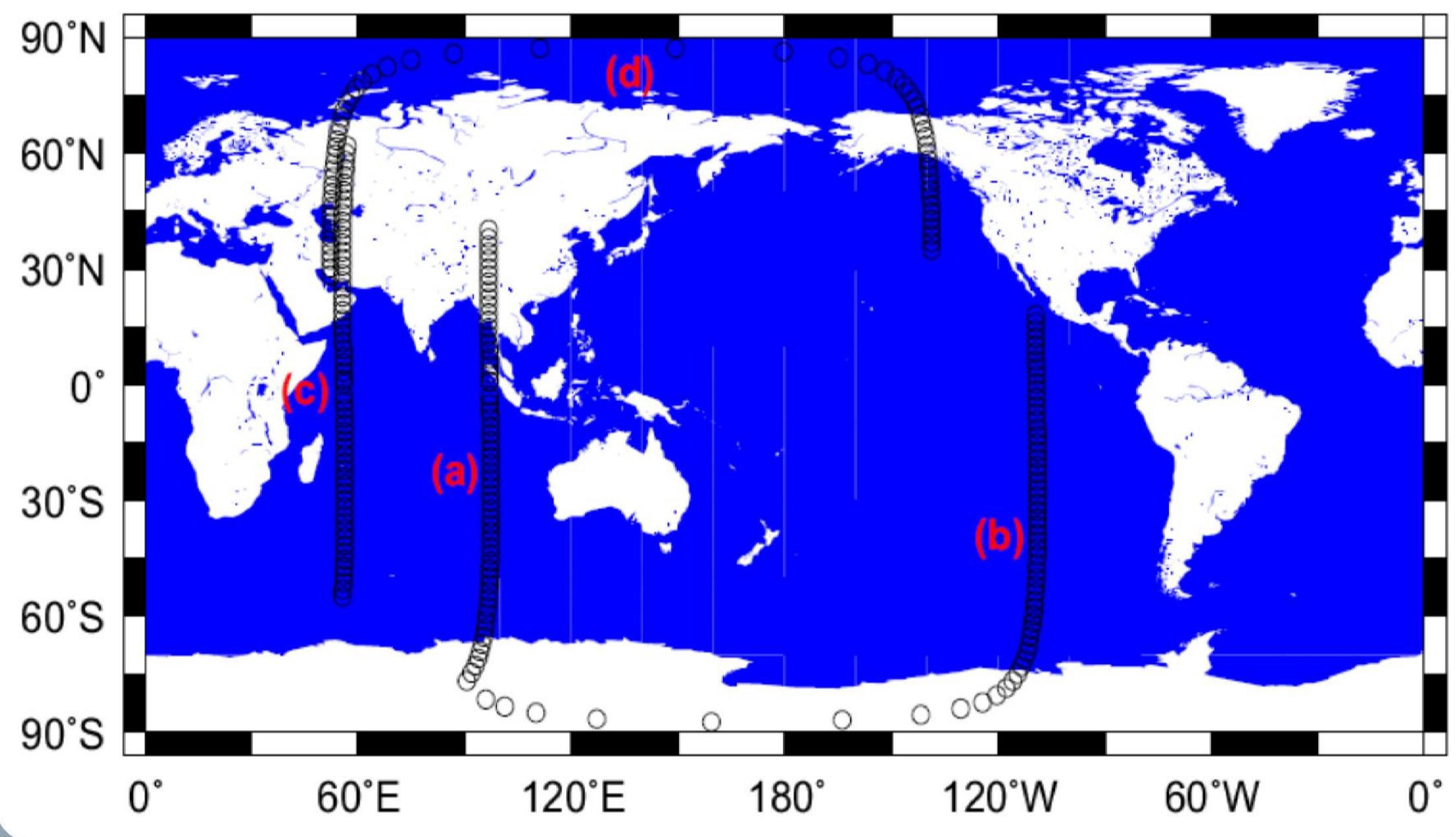

Four short arcs (30 min.) ground track of CHAMP 


\section{Kinematical POD - short arc case (b)}

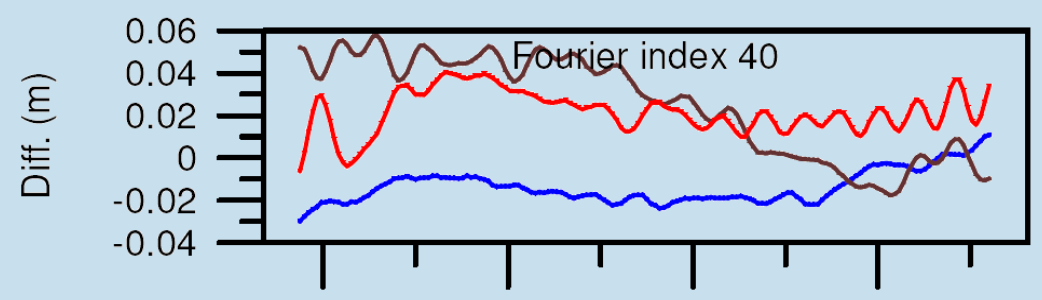

52475.53552475 .5452475 .54552475 .55 MJD (days)

\section{IGG - GFZ positions}

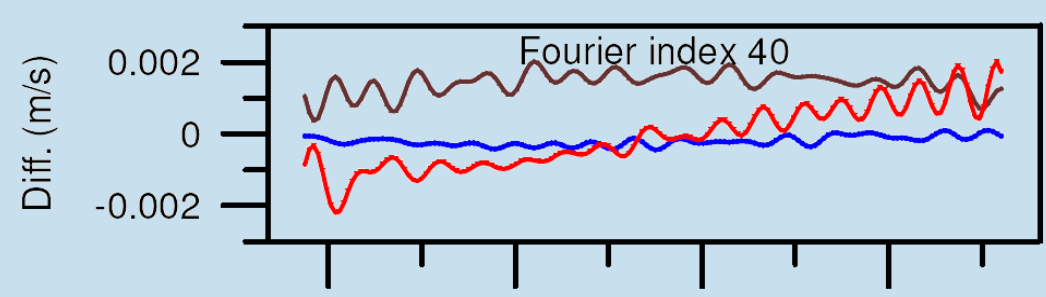

52475.53552475 .5452475 .54552475 .55 MJD (days)

IGG - GFZ velocities

RMS

\begin{tabular}{|c|c|c|}
\hline index & Pos. $(m)$ & Vel. $(m / s)$ \\
\hline 20 & 0.0503 & 0.0019 \\
\hline 30 & 0.0455 & 0.0018 \\
\hline 40 & 0.0449 & 0.0017 \\
\hline 59 & 0.0449 & 0.0017 \\
\hline
\end{tabular}

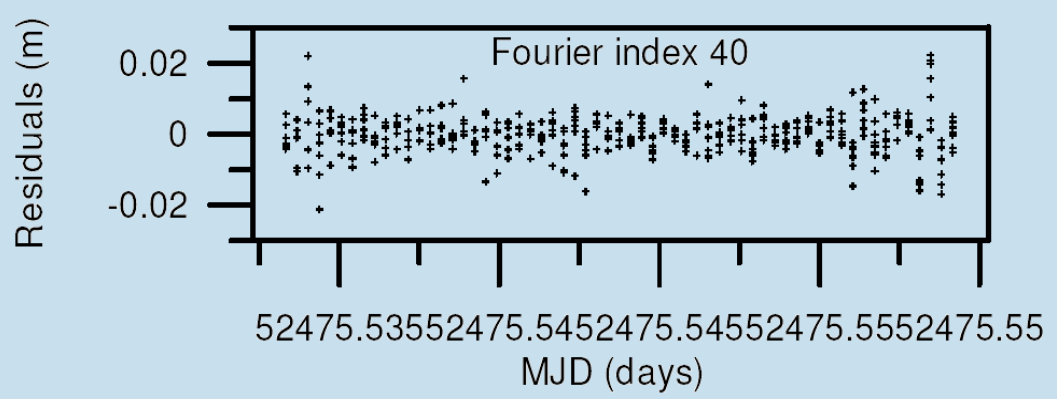

Statistical values

GPS-SST residuals 


\section{Processing procedures}

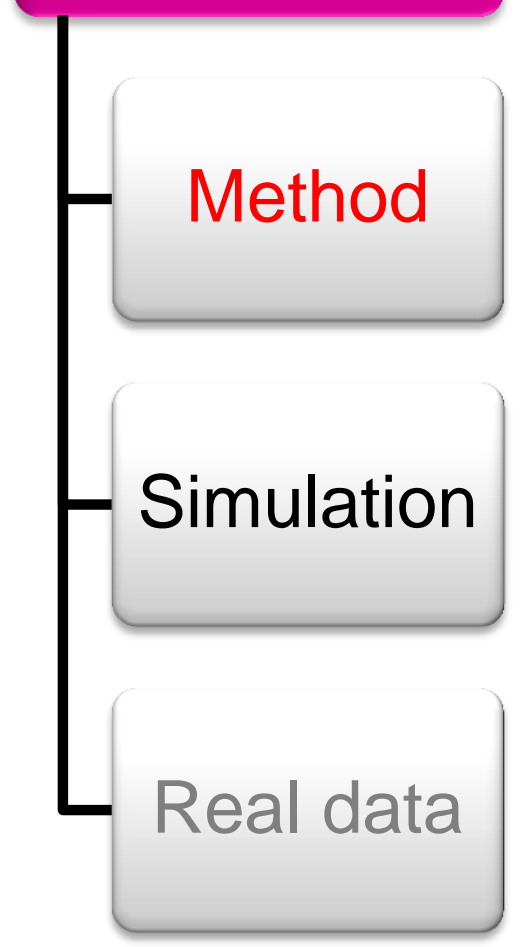




\section{Reduced-Kinematical POD - method}

$$
\begin{aligned}
& \begin{array}{c}
\text { Dynamical } \\
\text { info. }
\end{array} \\
& \left.\tilde{\mathbf{d}}_{v}=-\frac{2 T^{2}}{v^{2} \pi^{2}-\mu^{2}} \int_{\tau^{\prime}=0}^{1} \sin (v \pi \tau)+\sum_{v=1}^{n} \mathbf{d}_{v} \sin (v \pi \tau) ; \mathbf{r}, \dot{\mathbf{r}}\right) d \tau^{\prime}
\end{aligned}
$$

$\checkmark$ Introduction of an approximate force function $\left(\tilde{\mathbf{d}}_{i} \cdots \tilde{\mathbf{d}}_{j}\right), \mathbf{C}_{\left(\tilde{\mathbf{d}}_{i} \cdots \tilde{\mathbf{d}}_{j}\right)}$

$\checkmark$ Fixing only some orbit parameters $\left(\tilde{\mathbf{d}}_{i} \cdots \tilde{\mathbf{d}}_{j}\right), \mathbf{C}_{\left(\tilde{\mathbf{d}}_{i} \cdots \tilde{\mathbf{d}}_{j}\right)} \rightarrow \mathbf{0}$

$\checkmark$ Down- or up weighting $\mathbf{C}_{\left(\tilde{\mathbf{d}}_{1} \cdots \tilde{\mathbf{d}}_{n}\right)}$ in relation to $\mathbf{C}_{\left(\mathbf{d}_{1} \cdots \mathbf{d}_{n}\right)}$ 
Kinematical observation equation

Constraints

$\mathbf{I}_{1}=\left(\begin{array}{ll}\mathbf{A}_{1} & \mathbf{A}_{2}\end{array}\right)\left(\begin{array}{l}\mathbf{x}_{1} \\ \mathbf{x}_{2}\end{array}\right), \quad \mathbf{C}_{1}$

$\mathbf{I}_{2}=\left(\begin{array}{ll}\mathbf{0} & \mathbf{I}\end{array}\right)\left(\begin{array}{l}\mathbf{x}_{1} \\ \mathbf{x}_{2}\end{array}\right), \quad \mathbf{C}_{2}$

\section{凤}

凤

$$
\left(\begin{array}{c}
\hat{\mathbf{x}}_{1} \\
\hat{\mathbf{x}}_{2}
\end{array}\right)=\mathbf{N}^{-1}\left(\begin{array}{c}
\mathbf{A}_{1}^{\mathrm{T}} \mathbf{C}_{1}^{-1} \mathbf{I}_{1} \\
\mathbf{A}_{2}^{\mathrm{T}} \mathbf{C}_{1}^{-1} \mathbf{I}_{1}+\mathbf{C}_{2}^{-1} \mathbf{I}_{2}
\end{array}\right), \mathbf{N}^{-1}=\left(\begin{array}{ll}
\mathbf{Q}_{\hat{\mathbf{x}}_{1} \hat{\mathbf{x}}_{1}} & \mathbf{Q}_{\hat{\mathbf{x}}_{1} \hat{\mathbf{x}}_{2}} \\
\mathbf{Q}_{\hat{\mathbf{x}}_{2} \hat{\mathbf{x}}_{1}} & \mathbf{Q}_{\hat{\mathbf{x}}_{2} \hat{\mathbf{x}}_{2}}
\end{array}\right)
$$




\section{Processing procedures}

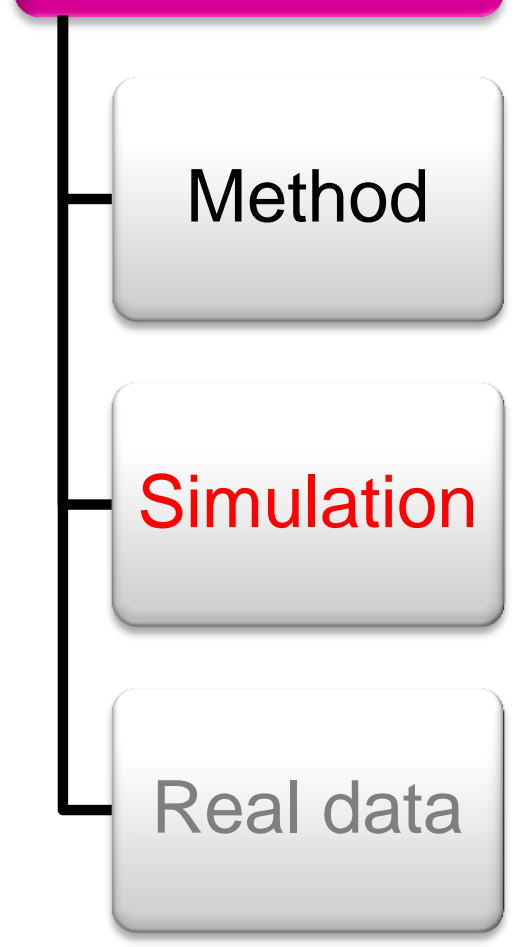




\section{RKPOD - simulation (Amp. 1-5, 2cm, N=300)}

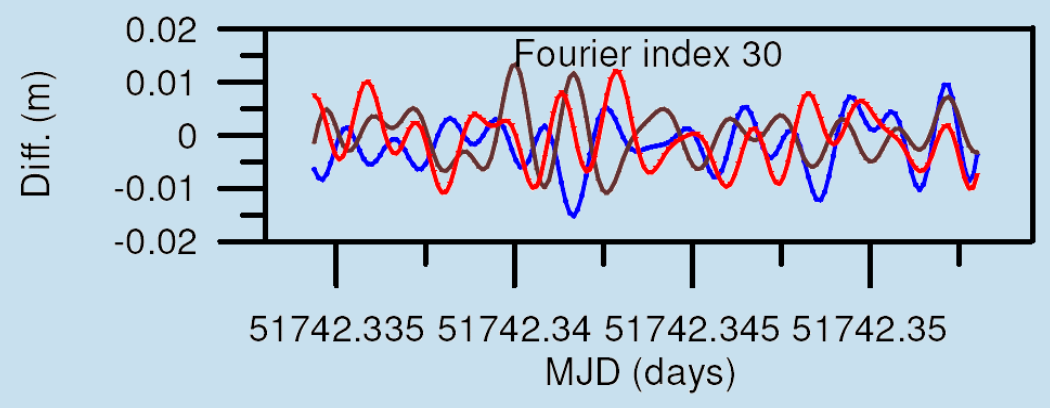

Position differences

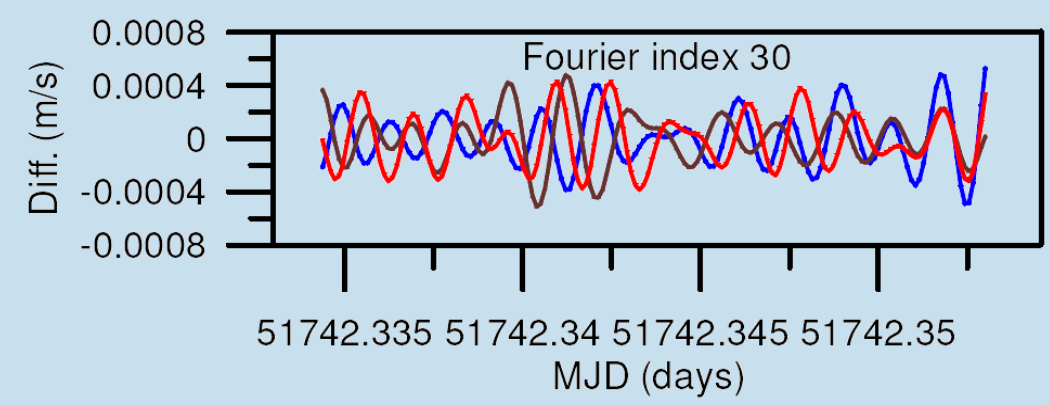

Velocity differences

RMS

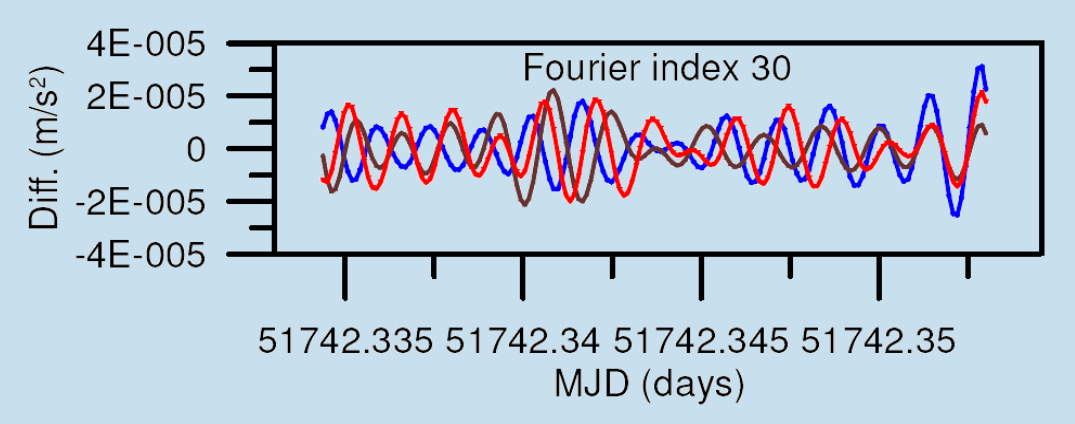

Acceleration differences

\begin{tabular}{|c|c|c|c|}
\hline index & Pos. $(m)$ & Vel. $(\mathrm{m} / \mathrm{s})$ & Acc. $\left(\mathrm{m} / \mathrm{s}^{2}\right)$ \\
\hline 20 & 0.012831 & 0.000316 & 0.000012 \\
\hline 30 & 0.008873 & 0.000337 & 0.000016 \\
\hline 40 & 0.014034 & 0.000402 & 0.000021 \\
\hline 59 & 0.011553 & 0.000721 & 0.000056 \\
\hline
\end{tabular}

\section{Statistical values}




\section{Conclusions and recommendations}

GNSS-LEO satellites configuration and geometrical strength play an important role in $\mathrm{POD}$,

- Kinematical POD can be used to recover the Earth's gravity field model based on the POD methods,

- No gravity field and no force models have been used in the Geometrical and Kinematical modes (advantage),

The proposed kinematical orbit determination method is very flexible. A smooth transition from kinematical to reduced kinematical and finally dynamical or vice-versa is possible. 


\section{Thank you for your attention}

